\title{
43. THE GALACTIC DISTRIBUTION OF YOUNG CEPHEIDS
}

\author{
G. A. TAMMAN N \\ Astronomisches Institut der Universität Basel, Binningen, Switzerland
}

\begin{abstract}
The distribution of long-period Population I cepheids is studied. An age comparison of cepheids and galactic clusters shows that cepheids with periods $>11.25 \mathrm{~d}$, corresponding to ages of $\lesssim 30 \times 10^{6} \mathrm{yr}$, should be almost as good spiral arm tracers as galactic clusters with earliest types b 2-3. The distances of cepheids are determined from a revised period-luminosity-colour relation and from colour excesses, for the determination of which a new, purely photometric method is given. The resulting distribution of cepheids shows a good correlation with the spiral arms, as traced by young clusters.
\end{abstract}

\section{Introduction}

Spiral arm tracers have to fulfill two requirements: they must be young enough to be typical for the spiral arm population and it must be possible to determine reliable distances for them. Next to the young galactic clusters and $\mathrm{H}$ II regions the Population I cepheids with long periods seem most suitable for this purpose. As for the clusters and exciting stars of $\mathrm{HII}$ regions the theory of stellar evolution provides age estimates for the cepheids, and their distances can well be determined from the period-luminosity-colour $(P-L-C)$ relation.

It is then of great interest to decide whether galactic clusters and $\mathrm{H}$ II regions on the one side and cepheids on the other side of comparable age define the same spiral arms. The contrary would be most severe: it would mean that there are separate places for stellar formation for different kinds of objects. It would seem easiest to solve the problem in extragalactic spirals whether the loci of young galactic clusters and young cepheids coincide. However, at present there are no complete surveys of cepheids in nearby spirals (M 31, M33) available. The detection of cepheids in more distant galaxies is so badly hampered by the discovery chance that a meaningful discussion of their distribution is impossible. It remains only the possibility to study the distribution of galactic cepheids and to compare it with the spiral arms as defined by galactic clusters and $\mathrm{H}$ II regions.

The study of the galactic distribution of cepheids has not yet led to clear results, mainly due to the paucity of long period cepheids. Their space density within $1500 \mathrm{pc}$ from the sun is roughly ten times lower than that of young galactic clusters, and their small number does not allow to outline spiral arms with any certainty. Therefore the question whether they are confined to spiral arms can only be settled, if their location is compared with more frequent spiral arm tracers as the young galactic clusters and Hil regions are (Becker, 1963; Becker and Fenkart, 1963). Kraft and Schmidt (1963) have found a vague correlation between bright cepheids and OB-associations, and Kraft (1963) a reasonable correspondance between bright cepheids and young clusters, which was confirmed by Schmidt-Kaler (1964) and by Tammann (1968) on the basis of an extended observational material. However, Fernie (1968) has recently 
denied this correlation; and he has found - not discriminating against cepheids with shorter periods - even an anti-correlation for cepheids with early B stars, galactic clusters, and $\mathrm{H}$ il regions.

Becker's (1969a) updated list of cluster and H II region distances and additional observations for long period cepheids as well as a new calibration of the slope and the zero point of the $P-L-C$ relation (Sandage and Tammann, 1969) and a better understanding of the age of cepheids as a function of period (Kippenhahn and Smith, 1969) make a new intercomparison desirable.

\section{The Age of Cepheids}

Kippenhahn and Smith (1969) have found from cepheids in galactic clusters an empirical relation between age and period. The relation predicts with surprising probability the age of a cepheid, in spite of the fact that the massive cepheids cross the instability strip several times and hence have different ages at a given period. The spread of age is offset by the fact, that the crossing times are quite different, and that the second crossing is by far the slowest. A least square solution of the data by Kippenhahn and Smith and giving proper weight to the individual crossing times leads to (Tammann, 1969a)

$$
\log t\left(\text { in } 10^{7} \mathrm{yr}\right)=1.16-0.651 \log P(\text { in } \mathrm{d}) .
$$

The maximum age of cepheids which are expected to outline well the spiral arms could be determined by Equation (1) and compared to the age of clusters with earliest main sequence type b2-3 $\left(U-B \lesssim-0^{\mathrm{m}} .80\right)$ or earlier, which are known to be spiral arm tracers by Becker's work. But the numerical age of clusters is critically dependent on the intrinsic colour of the brightest unevolved stars and hence on the colour excess assumed. It seems therefore safer to compare directly the periods with the spectral type of the earliest unevolved cluster members.

In Figure 1 the known cluster cepheids (Sandage and Tammann, 1969) are plotted with their periods against the earliest spectral types of their parent clusters. The four cepheids in the $\mathrm{h}+\chi$-association are plotted as an error box, because the spread in their periods as well as the work on the association by Wildey (1964) and Schild (1967) seem to indicate a finite formation time of the association. Also included in the diagram are WZ Sgr, SZ Tau, and Anon. Sct, which are believed to be members of the Sgr OB4-association (Tammann, 1969a), of NGC 1647 (Becker, 1969b), and of NGC 6649 (Tammann, 1969b), respectively. There is a clear relation between period and earliest spectral type. The scatter in the diagram is explainable by the non-unique relation between period and cepheid age, as already mentioned, and by observational errors in the determination of the earliest spectral types.

From Figure 1 one finds that all cepheids with $P>20 \mathrm{~d}$ and practically all cepheids with $P>15$ d should be as young or younger than b 2-3 clusters and hence be as good spiral arm tracers as the latter. To go to cepheids with periods as low as $11.25 \mathrm{~d}$ (average age $=3 \times 10^{7} \mathrm{yr}$ according to Equation (1)) would apparently mean to 


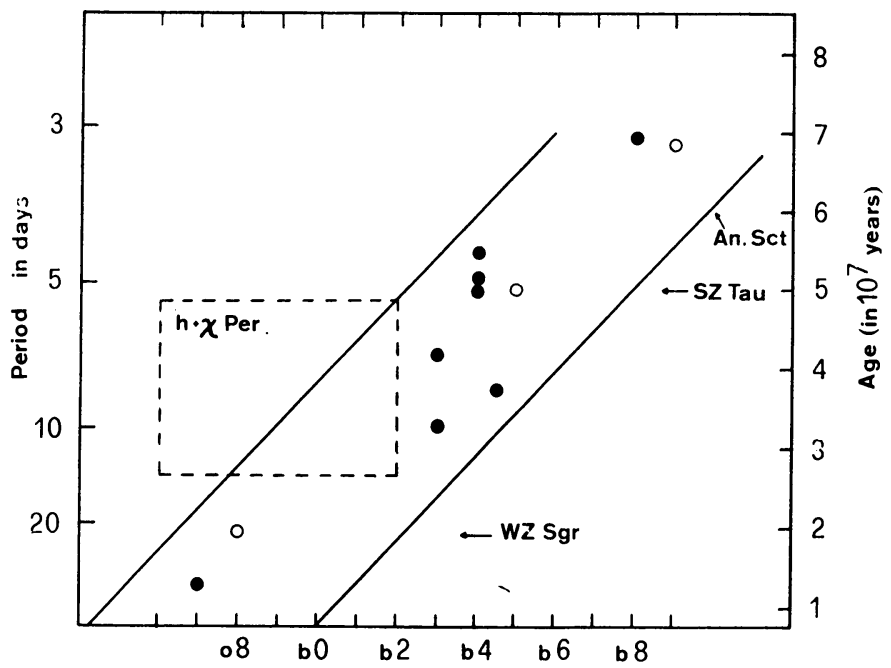

Fig. 1. The period of cluster cepheids vs. the earliest spectral type of their parent clusters. The scale at the right side gives the age of the cepheids according to Equation (1). Four cepheids in the $h+\chi$-association fall into the dotted square. Three cepheids, whose cluster membership is less reliable, are shown as open circles.

include some cepheids as old as b3-clusters which are known not to be confined to spiral arms.

\section{The P-L-C Relation}

The $P-L-C$ relation used here is:

$$
M_{\langle V\rangle}=-3.534 \log P+2.647\left(\left\langle B^{0}\right\rangle-\left\langle V^{0}\right\rangle\right)=2.469
$$

in the range $0.4<\log P<1.9$. This is essentially the same form as derived by Sandage and Tammann (1969) from 13 cepheids with known distances except for two slight modifications:

(a) The change of the pulsation constant $Q$ along a constant period line in the $\left(M_{V}, B-V\right)$-plane was considered by Sandage and Tammann (1969) but not taken into account. The best straight line approximation for this change follows from Christy's theory of stellar pulsation (Christy, 1968), which predicts:

$$
R^{1.69} / \mathfrak{M}^{0.66}=\text { const. (for } P<10 \mathrm{~d} \text { ) }
$$

and

$$
R^{1.83} / \mathfrak{M}^{0.78}=\text { const. }(\text { for } P>10 \mathrm{~d}) \text {. }
$$

Using a mass-luminosity relation of $L \propto \mathfrak{M}^{3.3}$ (Sandage and Gratton, 1963) and the same equations for the bolometric corrections and for the $\left(T_{\mathrm{e}}, B-V\right)$-relation of supergiants as in Sandage and Tammann (1968) one finds for constant period a relation of:

$$
M_{V}=2.647(B-V)-\text { const } .
$$


The coefficient of $(B-V)$ is the mean of 2.614 and 2.680 , which are the correct values for $P<10 \mathrm{~d}$ and $P>10 \mathrm{~d}$, respectively. (The value assumed before is 2.52 ).

(b) The mean magnitudes $\langle V\rangle$ and $\langle B\rangle$ of the cluster cepheids are taken from a list of photometric parameters, which were derived by Fourier analysis from all photoelectric observations of cepheids available (Schaltenbrand and Tammann, 1969)

The least square solution leading to Equation (2) is very little changed if EV Sct and VY Per are excluded ( 0.03 in the constant term). It is still somewhat doubtful if the parent cluster of EV Sct, NGC 6664, is real (Tammann, 1969c), and VY Per shows the greatest deviation and may not belong to the $h+\chi$-association. The average deviation from Equation (2) is for the 13 cepheids \pm 0 m.071 (without VY Per \pm 0 m 055 ), which is somewhat less than in the previous paper (Sandage and Tammann, 1969).

According to the theory of the instability strip the period-luminosity relation is depending on the effective temperature $T_{e}$, too. This dependence has usually been expressed by the observable quantity $(\langle B\rangle-\langle V\rangle)$. It is evident that instead of $(\langle B\rangle-\langle V\rangle)$ the colour $(\langle U\rangle-\langle B\rangle)$ could be used as well, the latter being almost as sensitive for temperature changes in supergiants. It is therefore attempted here to derive a $P-L-C$ relation in $M_{V}, \log P$, and $(\langle U\rangle-\langle B\rangle)$.

For this purpose 41 cepheids with complete UBV-photometry are taken from the list by Sandage and Tammann (1968). Reliable colour excesses are known for these mainly from the work by Kraft (1961) and Bahner et al. (1962); (the cepheids BY Cas, S Nor, and SV Per, which are suspected to have companions, are exluded as well as the peculiar cepheid Y Oph (Evans, 1968)). Mean magnitudes of these cepheids are taken from Schaltenbrand and Tammann (1969). The absolute magnitudes $M_{\langle V\rangle}$ are determined from Equation (2). The intrinsic colours $\langle U\rangle^{0}-\langle B\rangle^{0}$ are derived under the assumption $E_{U-B} / E_{B-V}=0.80$. By least square solution one then finds:

$$
M_{\langle V\rangle}=-3.382 \log P+1.834\left(\left\langle U^{0}\right\rangle-\left\langle B^{0}\right\rangle\right)-1.700 \text {. }
$$

The fact that similar solutions are found if the cepheids are devided into two groups with $E_{B-V}<0$ m.55 and $E_{B-V}>0$ m 55 , respectively, seems to prove in favour of the near correctness of the adopted ratio $E_{U-B} / E_{B-V}$. The average difference $\Delta M_{\langle V\rangle}$ between Equations (2) and (6) is for 41 cepheids \pm 0 m 094 .

\section{The Colour Excess of Cepheids}

The colour excesses of relatively few cepheids are well determined by means of spectroscopic or $\Gamma$-photometric observations. Since the colour excesses enter the distance determination of cepheids it is attempted here to derive the $E_{B-V}$-values purely from photometric data.

Equations (2) and (6) can be written in the form

$$
M_{\langle V\rangle}=3.534 \log P+2.647\left(\langle B\rangle-\langle V\rangle-E_{B-V}\right)-2.469
$$

and

$$
M_{\langle V\rangle}=3.382 \log P+1.834\left(\langle U\rangle-\langle B\rangle-0.80 E_{B-V}\right)-1.700 .
$$


Solving (7) and (8) for $E_{B-V}$ leads to:

$$
\begin{aligned}
E_{B-V}=-0.129 \log P+2.243(\langle B\rangle & -\langle V\rangle) \\
& -1.554(\langle U\rangle-\langle B\rangle)-0.652 .
\end{aligned}
$$

The colour excesses $E_{B-V}$ were determined from Equation (9) for 41 cepheids with known spectroscopic $E_{B-V}$-values. A comparison of the results of the two methods is shown in Figure 2. There is no indication for a systematic difference. The mean deviation from the $45^{\circ}$-line amounts to $\pm 0 \mathrm{~m} .08$. Since the average mean error of a spectroscopically determined colour excess is about \pm 0.03 , the colour excesses determined from Equation (9) should be good within \pm 0 m. 07 .

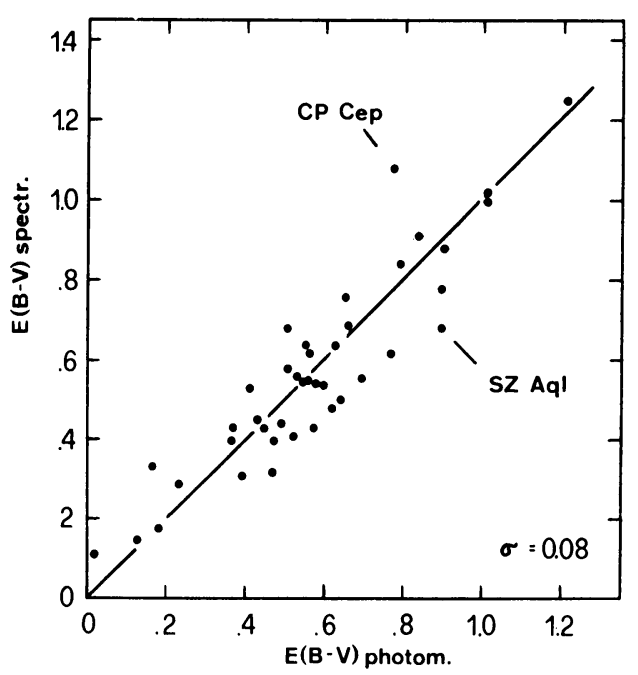

Fig. 2. Colour excesses $E_{B-V}$ from spectroscopic or $\Gamma$-photometric observations vs. $E_{B-V}$ from Equation (9). Two cepheids with exceptionally large deviations are indicated. The drawn line has the slope 1.

For cepheids where spectroscopic excesses are lacking as well as $U$-magnitudes $E_{B-V}$ was taken from the list by Fernie (1967). These values are derived mainly under the assumption that cepheids have the same intrinsic colours at maximum light, which seems to be correct within about \pm 0 . 10 (Sandage and Tammann, 1968). The fact that Fernie's intrinsic colours are systematically redder by $\sim 0$. 06 than those by Kraft can be neglected in the present context.

\section{The Distances of Cepheids}

From Equation (2) and an adopted absorption law $A_{V}=3.0 E_{B-V}$ the distance modulus becomes:

$$
\begin{aligned}
(m-M)^{0}=\langle V\rangle+3.534 \log P-2.647(\langle B\rangle-\langle V\rangle)- \\
-0.353 E_{B-V}+2.469 .
\end{aligned}
$$


There are few astronomical formulae so kindly deviced by nature as this one: the coefficients of the observed quantities are the greater the higher the precision is with which they can be observed. The only quantity where an observational error could have any effect on the distance modulus is $E_{B-V}$; even here an error of 0.10 affects the modulus by only 0.035 . The random scatter of cepheids around the adopted $P-L-C$ relation seems also to be quite small as indicated by the calibrating cepheids.

It remains the question whether systematic errors have entered the $P-L-C$ relation. The problem of the reliability of cluster distances based on the Hyades shall not be discussed here (see e.g. Sandage and Tammann, 1969), because any - quite unlikely change of the Hyades distance would equally affect the clusters and cepheids, whose distances are to be compared here. In determining the slope of the $P-L-C$ relation, RS Pup, the only calibrating cepheid with very long period, enters with great weight. However the slope found here seems to be quite reliable because in the range $0.4<$ $\log P<1.9$ it nearly coincides with the best linear fit to the ridge line of the $P-L-C$ relation derived from all well observed extragalactic cepheids (Sandage and Tammann, 1968) and agrees also very nearly with the slope Gascoigne (1969) found from the combined solution for cepheids in galactic clusters and in the Magellanic Clouds.

A list of distances from Equation (10) for cepheids with $P>9 \mathrm{~d}$ is given in Table I. It is attempted to include all galactic cepheids of Population I with sufficient observational data; however, cepheids of doubtful population and with suspected companions are excluded.

For a number of long period cepheids photoelectric photometry is missing. Distance estimates for these are taken from the list by Fernie and Hube (1968); they are not shown in Table I. The distances of cepheids in common in Table I and in the list by these authors agree reasonably well; this is somewhat surprising because they use a quite different period-luminosity relation.

\section{Galactic Distribution of Cepheids}

The distances of 32 cepheids with $P>15 \mathrm{~d}$ and of 20 cepheids with $15>P>11.25 \mathrm{~d}$ are shown in Figure 3 projected into the galactic plane; also drawn are 14 cepheids of the long period group and 7 of the shorter period group with lacking photoelectric observations. The young clusters and the HiI regions according to the latest results (Becker, 1969a) are drawn as open circles.

From Figure 3 the following conclusions can be drawn:

(a) The cepheids with $P>15 \mathrm{~d}$ either fall into the slightly extended boundaries, which are outlined as spiral arms by young cluster and $\mathrm{H}$ II regions, or lie at distances, where no young clusters and Hil regions are known. The same holds for 25 out of 27 cepheids with $15>P>11.25 \mathrm{~d}$, only 2 of them fall between reliably determined spiral arms (TT Aql and Z Sct.). This result conforms with the view that long period cepheids, young clusters and the exciting stars of $\mathrm{H}$ II regions were formed essentially at the same places.

(b) The exception of TT Aql is not surprising. It lies mid-way between the spiral 
TABLE I

Distances of Cepheids with $P>9 d$

\begin{tabular}{|c|c|c|c|c|c|c|c|c|c|c|}
\hline $\begin{array}{l}\text { (1) } \\
\text { Cepheid }\end{array}$ & $\begin{array}{l}\text { (2) } \\
l^{\text {II }}\end{array}$ & $\begin{array}{l}\text { (3) } \\
b^{\text {II }}\end{array}$ & $\begin{array}{l}(4) \\
\log P\end{array}$ & $\begin{array}{l}(5) \\
\langle U\rangle\end{array}$ & $\begin{array}{l}(6) \\
\langle B\rangle\end{array}$ & $\begin{array}{l}\text { (7) } \\
\langle V\rangle\end{array}$ & $\begin{array}{l}(8) \\
E_{\mathrm{sp}}\end{array}$ & $\begin{array}{l}(9) \\
E_{\mathrm{ph}}\end{array}$ & $\begin{array}{l}(10) \\
E_{\text {Fernie }}\end{array}$ & $\begin{array}{l}(11) \\
r(\mathrm{pc})\end{array}$ \\
\hline SZ Aql & 35.6 & -2.3 & 1.234 & 11.06 & 10.09 & 8.66 & 0.68 & & & 1950 \\
\hline TT Aql & 36.0 & -3.1 & 1.138 & 9.44 & 8.44 & 7.14 & 0.55 & & & 1000 \\
\hline FN Aql & 38.5 & -3.1 & 0.977 & 10.53 & 9.62 & 8.38 & & 0.59 & & 1450 \\
\hline RX Aur & 165.8 & -1.3 & 1.065 & 9.33 & 8.65 & 7.68 & & 0.32 & & 1760 \\
\hline SY Aur & 164.7 & +2.1 & 1.006 & 10.83 & 10.12 & 9.06 & 0.44 & & & 2660 \\
\hline YZ Aur & 167.3 & +0.9 & 1.260 & 12.74 & 11.79 & 10.38 & & 0.87 & & 4480 \\
\hline AN Aur & 164.9 & -1.0 & 1.012 & 12.49 & 11.66 & 10.44 & & 0.68 & & 4010 \\
\hline RW Cam & 144.9 & +3.8 & 1.215 & - & 10.06 & 8.67 & & & 0.70 & 1990 \\
\hline SS CMa & 239.2 & -4.2 & 1.092 & 11.97 & 11.11 & 9.87 & & 0.62 & & 3510 \\
\hline$l \mathrm{Car}$ & 283.2 & -7.0 & 1.551 & 6.04 & 5.00 & 3.72 & & 0.39 & & 430 \\
\hline U Car & 289.1 & +0.1 & 1.588 & 8.35 & 7.46 & 6.27 & & 0.43 & & 1630 \\
\hline VY Car & 286.6 & +1.2 & 1.278 & 9.36 & 8.58 & 7.45 & & 0.51 & & 1790 \\
\hline WZ Car & 289.3 & -1.2 & 1.362 & - & 10.46 & 9.29 & & & 0.33 & 4730 \\
\hline XX Car & 291.3 & -4.9 & 1.196 & - & 10.39 & 9.35 & & & 0.30 & 4290 \\
\hline XY Car & 291.4 & -3.9 & 1.095 & - & 10.50 & 9.30 & & & 0.49 & 2880 \\
\hline XZ Car & 290.3 & -0.8 & 1.221 & - & 9.84 & 8.59 & & & $0.55^{*}$ & 1680 \\
\hline YZ Car & 285.6 & -1.4 & 1.259 & 10.63 & 9.83 & 8.71 & & 0.45 & & 3160 \\
\hline AQ Car & 285.8 & -3.3 & 0.990 & 10.44 & 9.78 & 8.84 & & 0.29 & & 2800 \\
\hline CR Car & 285.7 & -0.4 & 0.990 & 13.82 & 12.90 & 11.58 & & 0.75 & & 5750 \\
\hline FI Car & 287.8 & +0.7 & 1.129 & - & 13.24 & 11.65 & & & 0.88 & 5170 \\
\hline FO Car & 290.5 & -2.1 & 1.015 & - & 12.05 & 10.78 & & & 0.64 & 4440 \\
\hline FR Car & 291.1 & +0.6 & 1.030 & 11.65 & 10.82 & 9.68 & & 0.51 & & 3260 \\
\hline RW Cas & 129.0 & -4.6 & 1.170 & 11.41 & 10.46 & 9.21 & 0.41 & & & 2980 \\
\hline RY Cas & 115.3 & -3.3 & 1.084 & 12.34 & 11.31 & 9.94 & 0.76 & & & 2980 \\
\hline SZ Cas & 134.8 & -1.2 & 1.134 & 12.39 & 11.32 & 9.83 & 0.88 & & & 2546 \\
\hline $\mathrm{CH}$ Cas & 112.9 & +1.6 & 1.179 & - & 12.61 & 10.96 & & & 0.94 : & 3830 \\
\hline CY Cas & 113.9 & +2.0 & 1.158 & - & 13.35 & 11.65 & 1.10 & & & 4640 \\
\hline DD Cas & 116.8 & +0.5 & 0.992 & 12.00 & 11.08 & 9.86 & 0.56 & & & 3020 \\
\hline TX Cen & 315.2 & -0.6 & 1.233 & 13.50 & 12.23 & 10.53 & & 1.04 & & 3130 \\
\hline VW Cen & 307.6 & -1.6 & 1.177 & 12.53 & 11.57 & 10.22 & & 0.73 & & 4010 \\
\hline XX Cen & 309 & +4.6 & 1.040 & 9.47 & 8.79 & 7.82 & & 0.34 & & 1790 \\
\hline KK Cen & 294.2 & +2.7 & 1.086 & - & 12.82 & 11.50 & & & 0.57 & 6620 \\
\hline KN Cen & 307.8 & -2.1 & 1.532 & 12.50 & 11.40 & 9.84 & & 0.96 & & 4430 \\
\hline V339 Cen & 313.5 & -0.5 & 0.976 & 10.73 & 9.90 & 8.70 & & 0.64 & & 1740 \\
\hline CP Cep & 100.4 & +1.1 & 1.252 & 13.53 & 12.18 & 10.54 & 1.08 & & & 3480 \\
\hline SU Cru & 299.2 & -0.6 & 1.109 & 12.82 & 11.56 & 9.79 & & 1.21 & & 1640 \\
\hline X Cyg & 76.9 & -4.3 & 1.215 & 8.43 & 7.55 & 6.40 & 0.45 & & & 970 \\
\hline SZ Cyg & 84.4 & +4.0 & 1.179 & 12.19 & 10.95 & 9.42 & & 0.70 & & 2250 \\
\hline TX Cyg & 84.4 & -2.3 & 1.168 & 12.77 & 11.37 & 9.49 & 1.25 & & & 1380 \\
\hline VX Cyg & 82.2 & -3.5 & 1.304 & 13.18 & 11.81 & 10.07 & & 0.96 & & 2730 \\
\hline BZ & 84. & +1.4 & 1.0 & 12. & 11. & 10.22 & 1.00 & & & 2140 \\
\hline CD Cyg & 71.1 & +1.4 & 1.232 & 11.33 & 10.29 & 8.97 & 0.64 & & & 2580 \\
\hline EZ Cyg & 67.1 & +0.6 & 1.067 & 13.70 & 12.51 & 11.06 & & 0.63 & & 4420 \\
\hline
\end{tabular}


Table I (continued)

\begin{tabular}{|c|c|c|c|c|c|c|c|c|c|c|}
\hline Cepheid & $l^{1 \mathrm{I}}$ & $b^{\mathrm{II}}$ & $\log P$ & $\langle\boldsymbol{U}\rangle$ & $\langle\boldsymbol{B}\rangle$ & $\langle\boldsymbol{V}\rangle$ & $E_{\mathrm{sp}}$ & $E_{\mathrm{ph}}$ & $E_{\text {Fernie }}$ & $r(\mathrm{pc})$ \\
\hline$\beta$ Dor & 271.7 & -32.8 & 0.993 & 5.13 & 4.56 & 3.75 & & 0.14 & & 320 \\
\hline$\zeta \mathrm{Gem}$ & 195.7 & +11.9 & 1.007 & 5.30 & 4.71 & 3.89 & 0.15 & & & 350 \\
\hline AA Gem & 184.6 & +2.7 & 1.053 & 11.56 & 10.81 & 9.71 & & 0.53 & & 3600 \\
\hline Z Lac & 105.8 & -1.6 & 1.037 & 10.33 & 9.58 & 8.43 & 0.48 & & & 1860 \\
\hline T Mon & 203.6 & -2.6 & 1.432 & 8.28 & 7.33 & 6.14 & 0.43 & & & 1180 \\
\hline SV Mon & 203.7 & -3.7 & 1.183 & 10.11 & 9.30 & 8.25 & & 0.28 & & 2540 \\
\hline S Mus & 299.6 & -7.5 & 0.985 & 7.52 & 6.96 & 6.14 & & 0.22 & & 920 \\
\hline UU Mus & 296.8 & -3.2 & 1.066 & 11.84 & 10.91 & 9.78 & & 0.29 & & 3840 \\
\hline S Nor & 327.8 & -5.4 & 0.989 & 8.01 & 7.36 & 6.41 & 0.21 & & & 910 \\
\hline U Nor & 325.6 & -0.2 & 1.102 & 11.96 & 10.83 & 9.23 & & 1.04 & & 1570 \\
\hline SY Nor & 327.5 & -0.7 & 1.102 & 11.72 & 10.85 & 9.50 & & 0.89 & & 2470 \\
\hline SV Per & 162.6 & -1.5 & 1.047 & 10.55 & 9.99 & 8.95 & 0.44 & & & 2800 \\
\hline VX Per & 132.8 & -3.0 & 1.037 & 11.38 & 10.52 & 9.30 & & 0.61 & & 2500 \\
\hline X Pup & 236.1 & -0.8 & 1.414 & 10.70 & 9.76 & 8.54 & & 0.45 & & 3350 \\
\hline RS Pup & 252.4 & -0.2 & 1.617 & 9.54 & 8.44 & 7.01 & & 0.65 & & 1710 \\
\hline VZ Pup & 243.4 & -3.3 & 1.365 & 11.56 & 10.77 & 9.61 & & 0.57 & & 5290 \\
\hline AD Pup & 241.9 & -0.0 & 1.133 & - & 10.97 & 9.88 & & & $0.42^{*}$ & 4620 \\
\hline AQ Pup & 246.2 & +0.1 & 1.475 & 11.09 & 10.15 & 8.79 & 0.62 & & & 3370 \\
\hline VY Sgr & 10.1 & -1.1 & 1.132 & - & 13.50 & 11.53 & & & $1.30^{*}$ & 2920 \\
\hline WZ Sgr & 12.1 & -1.3 & 1.339 & 10.56 & 9.41 & 8.02 & 0.68 & & & 1820 \\
\hline YZ Sgr & 17.8 & -7.1 & 0.980 & 9.13 & 8.36 & 7.34 & & 0.32 & & 1230 \\
\hline RY Sco & 356.5 & -3.4 & 1.308 & 10.49 & 9.44 & 7.99 & & 0.79 & & 1560 \\
\hline V500 Sco & 359.0 & -1.4 & 0.969 & 11.03 & 10.03 & 8.74 & & 0.55 & & 1610 \\
\hline Y Sct & 24.0 & -0.9 & 1.015 & 12.29 & 11.19 & 9.63 & 0.80 & & & 1810 \\
\hline Z Sct & 26.8 & -0.8 & 1.111 & 11.88 & 10.94 & 9.60 & & 0.74 & & 2760 \\
\hline RU Sct & 28.2 & +0.2 & 1.294 & 12.61 & 11.21 & 9.50 & & 0.84 & & 2200 \\
\hline TY Sct & 28.1 & +0.1 & 1.044 & 13.80 & 12.52 & 10.79 & & 1.11 & & 2490 \\
\hline UZ Sct & 19.2 & -1.5 & 1.169 & 14.78 & 13.17 & 11.28 & & 0.94 & & 3220 \\
\hline RY Vel & 282.6 & +1.5 & 1.449 & 10.71 & 9.71 & 8.36 & & 0.64 & & 2690 \\
\hline RZ Vel & 262.9 & -1.9 & 1.310 & - & 8.23 & 7.11 & & & 0.30 & 1700 \\
\hline SV Vel & 286.0 & +2.4 & 1.149 & - & 9.65 & 8.57 & & & 0.35 & 2650 \\
\hline SW Vel & 266.2 & -3.0 & 1.371 & 10.13 & 9.28 & 8.13 & & 0.44 & & 2790 \\
\hline SX Vel & 265.5 & -2.2 & 0.980 & - & 9.14 & 8.26 & & & 0.33 & 2240 \\
\hline DD Vel & 271.5 & -1.4 & 1.120 & - & 14.13 & 12.47 & & & $0.98^{*}$ & 6860 \\
\hline DR Vel & 273.2 & +1.3 & 1.049 & - & 11.10 & 9.54 & & & 0.85 & 1810 \\
\hline Ex Vel & 274.1 & -2.2 & 1.122 & - & 13.31 & 11.72 & & & 0.88 & 5340 \\
\hline SV Vul & 63.9 & +0.3 & 1.654 & 9.85 & 8.69 & 7.22 & 0.64 & & & 1930 \\
\hline
\end{tabular}

Column 1-4: name of cepheid with galactic coordinates and logarithm of period.

Column 5-6: mean magnitudes from Schaltenbrand and Tammann (1969).

Column 8: $\quad E_{B-V}$ from spectroscopic or $\Gamma$-photometric observations (Sandage and Tammann, 1968).

Column 9: $\quad E_{B-v}$ from Equation (9).

Column 10: $E_{B-V}$ from Fernie (1967). Stars* indicate that $E_{B-V}$ was determined from the periodcolour relation for galactic cepheids (Sandage and Tammann, 1968, Equation (7)).

Column 11: the distance in $\mathrm{pc}$ from Equation (10). 


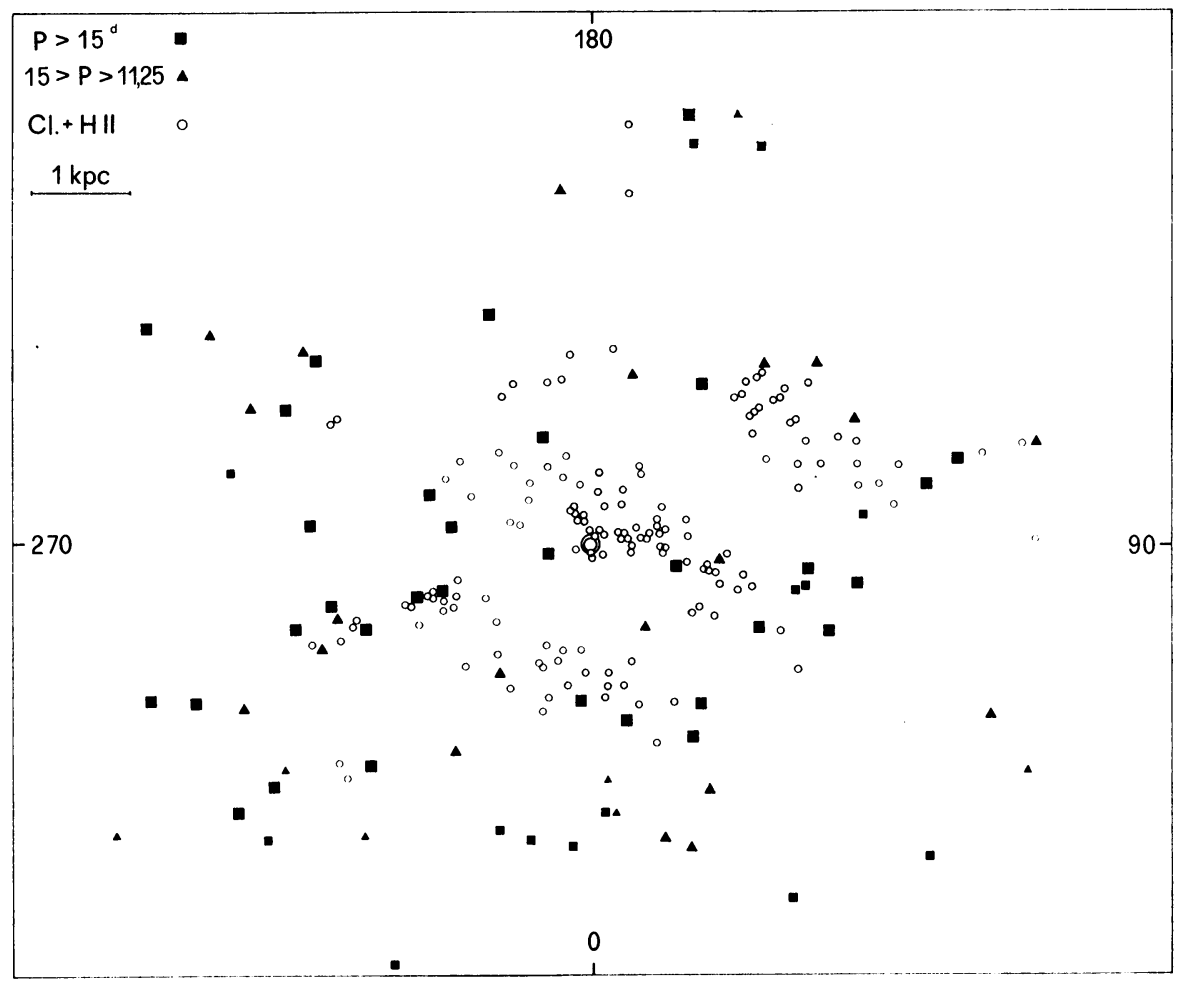

Fig. 3. The galactic distribution of young objects projected into the galactic plane. Big squares $(\square)$ : cepheids with $P>15$ days; big triangles $(\boldsymbol{\Lambda})$ : cepheids with $15>P>11.25$ days. Small squares $(\square)$ and small triangles $(\Delta)$ : cepheids with uncertain distances in the corresponding period intervals; open circles $(O)$ : clusters with earliest type b2-3 and HII regions (compare Becker and Fenkart, this volume, p. 205, Figure 1).

arms 0 and $-I$ (the designation of spiral arms according to Becker, (1963)). Its age is from Equation (1) $2.7 \times 10^{7} \mathrm{yr}$. If one assumes that it has moved in the galactic plane with 1.5 times the random speed of cepheids of $15 \mathrm{~km} \mathrm{~s}^{-1}$ (Kraft and Schmidt, 1963; Oort, 1964), and that its velocity is perpendicular to the axis of a spiral arm, it has travelled during its life-time very nearly the distance between the ridge line of one of the neighbouring spiral arms and its present location.

The case of Z Sct, lying between - I and - II, is less clear. There is a slight indication from Figure 3 that the distance between these two spiral arms decreases if one goes from $l^{\mathrm{II}}=310^{\circ}$ to $0^{\circ}$ to $30^{\circ}$. This possible merging of the spiral arms in the direction of Scutum might be supported by the distribution of early Be-stars (Schmidt-Kaler, 1964). If this was correct, $Z$ Sct would fit well into the spiral pattern.

(c) The agreement between young clusters, $\mathrm{H}_{\text {II }}$ regions and long period cepheids is especially tight in the spiral arm $-\mathrm{I}$; it is still quite reasonable for the spiral arms 0 and + I. The existence of the spiral arms - II and + II, barely indicated by young clusters and $\mathrm{H}$ II regions, seems to be confirmed by the cepheids. There is hope that 
the spiral arm - II can be traced quite well from cepheids, when photoelectric UBVobservations of all long period cepheids in this direction become available.

(d) If one goes to shorter periods $(P<11.25 \mathrm{~d})$ more and more cepheids fall inbetween the spiral arms. Eventually the correlation between young clusters, H II regions and cepheids is smeared out and turns into the anti-correlation found by Fernie (1968).

\section{Acknowledgements}

I would like to thank Prof. W. Becker for many discussions and for letting me use his unpublished list of data for the clusters and $\mathrm{H}_{\text {II }}$ regions. I am also indebted to Prof. R. F. Christy and Dr. S. C. B. Gascoigne for unpublished material. I acknowledge that part of this work was supported by the Swiss National Science Foundation.

\section{References}

Bahner, K., Hiltner, W. A., and Kraft, R. P.: 1962, Astrophys. J. Suppl. Ser. 6, 319.

Becker, W.: 1963, Z. Astrophys. 57, 117.

Becker, W.: 1969a, unpublished.

Becker, W.: 1969b, private communication.

Becker, W. and Fenkart, R. P.: 1963, Z. Astrophys. 56, 257.

Christy, R. F.: 1968, private communication.

Evans, T. L.: 1968, Monthly Notices Roy. Astron. Soc. 141, 109.

Fernie, J. D.: 1967, Astron. J. 72, 422.

Fernie, J. D.: 1968, Astron. J. 73, 995.

Fernie, J. D. and Hube, J. O.: 1968, Astron. J. 73, 492.

Gascoigne, S. C. B.: 1969, Monthly Notices Roy. Astron. Soc. 146, 1.

Kippenhahn, R. and Smith, L.: 1969, Astron. Astrophys. 1, 142.

Kraft, R. P.: 1961, Astrophys. J. 134, 616.

Kraft, R. P.: 1963, Stars and Stellar Systems 5, 157.

Kraft, R. P. and Schmidt, M.: 1963, Astrophys. J. 137, 249.

Oort, J. H.: 1964, IAU Symposium No. 20, p. 8.

Sandage, A. and Gratton, L.: 1963, Proc. Int. School of Physics "Enrico Fermi", Academic Press, New York, p. 11.

Sandage, A. and Tammann, G. A.: 1968, Astrophys. J. 151, 531.

Sandage, A. and Tammann, G. A.: 1969, Astrophys. J. 157, 683.

Schaltenbrand, R. and Tammann, G. A.: 1969, unpublished.

Schild, R.: 1967, Astrophys. J. 148, 449.

Schmidt-Kaler, Th.: 1964, Z. Astrophys. 58, 217.

Tammann, G. A.: 1968, Colloque Obs. Genève, May 16-18, 1968, chapter 18.

Tammann, G. A.: 1969a, Cepheiden als Mitglieder offener Sternhaufen und Sternassoziationen, Habilitationsschrift, Basel.

Tammann, G. A.: 1969b, Astron. Astrophys. 3, 308.

Tammann, G. A.: 1969c, IAU Inform. Bull. Variable Stars, No. 351.

Wildey, R. L.: 1964, Astrophys. J. Suppl. Ser. 8, 439. 